\title{
MODE COUPLING PHENOMENA IN LONG-PERIOD FIBER GRATINGS FORMED WITH MICRO BENDING
}

\author{
Chung-Yi Chiu, Su-Fang Chen, Yean-Woei Kiang, Hua-Kuang Liu and C. C. Yang \\ Graduate Institute of Electro-Optical Engineering, Graduate Institute of Communication \\ Engineering, and Department of Electrical Engineering, National Taiwan University, \\ 1, Roośevelt Road, Section 4, Taipei, Taiwan, R.O.C. \\ (phone) 886-2-23657624 (fax) 886-2-23652637 (e-mail) ccy@cc.ee.ntu.edu.tw \\ Kung-Jen Ma \\ Department of Mechanical Engineering, Chung Hua University, Hsinchu, Taiwan, R.O.C.
}

Abstract- Coupling with $H E_{2 \mu}$ modes resulted in a coupling coefficient variation period the same as that of
micro bending. However, the coupling with $H E_{l \mu}$ modes led to a period only one-half the micro bending period.

In a dynamic long-period fiber grating, implemented either with acoustic vibration or periodical loading, the major mechanism for the grating effects is micro bending. Although long-period fiber gratings with micro bending have been widely implemented, the theories behind their coupling phenomena have not been well studied yet. In this letter, we report the calculation results of the coupling behaviors between the core mode and various cladding modes in a fiber with micro bending. It was discovered that depending on the odd or even nature of a cladding mode, the coupling coefficient showed a longitudinal period either the same as or one-half the period of micro bending. Experimental results of double-sided loading-induced micro bending were used for reasonable comparison with the theoretical predictions. Figure 1 shows three sets of coupling coefficient data in the case of $10 \%$ bending. In other words, the fiber axis deviates from the un-perturbed situation by $10 \%$ of the core radius $\left(d=0.1 a_{l}\right)$. In portion (a) of this figure, the coupling coefficient between the core mode (HE $\mathrm{F}_{11}$ mode) and itself (counter-propagating) as a function of fiber length is shown. It is interesting to see that its period is only $350 \mu \mathrm{m}$, one-half the micro bending period. In portion (b), the coupling coefficients between the core mode and two cladding modes, $\mathrm{HE}_{12}$ and $\mathrm{HE}_{17}$, are shown. Again, they have the period of $350 \mu \mathrm{m}$. However, in potion (c), in which the coupling coefficients between the core and cladding modes, $\mathrm{HE}_{21}$ and $\mathrm{HE}_{22}$, are shown, the period of coupling coefficient of $\Lambda=700 \mu \mathrm{m}$ can be observed. Although the amplitudes of coupling coefficients in the coupling processes with $\mathrm{HE}_{2 \mu}$ modes are a few times larger than those with $\mathrm{HE}_{1 \mu}$ cladding modes, the later do exist and can produce significant effects in the coupling behaviors. The difference in the variation period of coupling coefficient between the processes with $\mathrm{HE}_{\mu \mu}$ and $\mathrm{HE}_{2 \mu}$ modes is due to the even and odd nature, respectively, of their mode field profiles. With the symmetric refractive index perturbation distributions between a bending maximum and its counterpart $0.5 \Lambda$ away, the coupling between two even modes $\left(\mathrm{HE}_{1 \mu}\right)$ results in the same coupling coefficient at these two points. Hence, their coupling coefficient variation shows a period of $0.5 \Lambda$ $(350 \mu \mathrm{m})$. On the other hand, for coupling between the core mode (even mode) and an odd cladding mode $\left(\mathrm{HE}_{2 \mu}\right)$, the coupling coefficient variation has a period of $\Lambda(700 \mu \mathrm{m})$. Such coupling behaviors are quite different from those in a fiber grating with refractive index variation in the core. Different coupling coefficient variation periods lead to different phase matching conditions.

In experiment, double-sided periodical loading was applied to a single -mode fiber for micro bending effects. Three major spectral depressions can be clearly observed with their minima around 1510 , 1555 and $1620 \mathrm{~nm}$. The transmission spectrum with the couplings between the core mode $\left(\mathrm{HE}_{11}\right)$ and all possible phase-matched cladding modes was calculated. The three major depression minima, which correspond to the effective couplings with $\mathrm{HE}_{17}, \mathrm{HE}_{21}$ and $\mathrm{HE}_{22}$ modes, are quite close to those of the experimental result. The relative depression depths agree well between the theoretical and experimental data. Although certain discrepancies existed, the theoretical predictions agreed reasonably well with the experimental data. The experimental results consisted of the coupling processes with coupling coefficient periods of $\Lambda$ and $0.5 \Lambda$.

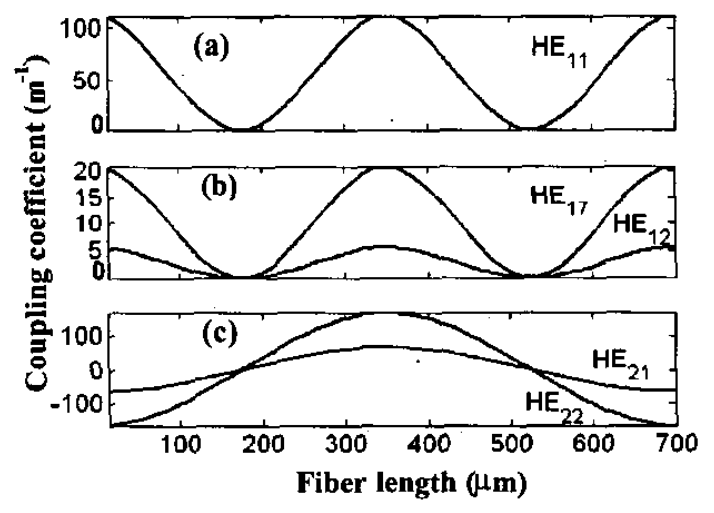

Fig. 1 Coupling coefficient variations along fiber. 